\title{
GEOMETRICAL IMPLICATION OF THE CME EARTHWARD DIRECTION PARAMETER AND ITS COMPARISON WITH CONE MODEL PARAMETERS
}

\author{
Y.-J. Moon ${ }^{1}$, R.-S. KIM ${ }^{2,3}$, \& K.-S. CHO ${ }^{2}$ \\ ${ }^{1}$ Department of Astronomy and Space Science, Kyung Hee University, Yongin 446-701, Korea \\ E-mail: moonyj@khu.ac.kr \\ ${ }^{2}$ Korea Astronomy and Space Science Institute, Whaamdong, Yooseong-ku, Daejeon, 305-348, Korea \\ ${ }^{3}$ Chungnam National University, Yooseong-ku, Daejeon, Korea
}

(Received March 3, 2009; Accepted April 14, 2009)

\begin{abstract}
Recently, we suggested a CME earthward direction parameter as an important geoeffective parameter that has been demonstrated by front-side halo CME data. In this study, we present the geometrical implication of this parameter by comparing with the parameters from a CME cone model. Major results from this study can be summarized as follows. First, we derive an analytic relationship between the cone model parameters (the half angular width of a cone and the angle between the cone axis and the plane of sky) and the earthward direction parameter. Second, we demonstrate a close relationship between the earthward direction parameter and the cone axis angle using 32 front-side full halo CMEs. Third, we found that there is noticeable inconsistency between the cone axis angles estimated from the cone model fitting to the CMEs and from their associated flare positions, implying that the flare position should not be considered as a good earthward direction parameter. Finally we present several advantages of our earthward direction parameter in terms of the forecast of a geomagnetic storm based on CME parameters.
\end{abstract}

Key words : Sun: coronal mass ejection

\section{INTRODUCTION}

Coronal mass ejections (CMEs) have been regarded as the main cause of heliospheric and geomagnetic disturbances. Front-side halo CMEs are the most potential candidate for producing geomagnetic storms. Recently, we have made a series of studies on the relationship between solar disturbances (CMEs or coronal shocks) and near-Earth disturbances (interplanetary shocks or geomagnetic storms): interplanetary shock propagation model (Moon et al. 2002a; Cho et al. 2003; Kim et al. 2007), CME geoeffectiveness depending on its speed and position (Kim et al. 2005), CME geoeffectiveness depending on its magnetic field orientation (Kang et al. 2006), and CME earthward direction as an important geoeffective parameter (Moon et al. 2005; Kim et al. 2008). Other aspects of CME geoeffectiveness are well reviewed in Gopalswamy et al.(2007) and Kim et al.(2008).

Moon et al. (2005) suggested a new geoeffective parameter for very fast halo CMEs. This parameter, which is called the earthward direction parameter DP, is defined as the ratio of the shortest to the longest distances of the CME front measured from the solar center. It is directly observable from coronagraph observations like SOHO LASCO. They showed that the direction parameter and the minimum Dst index have

Corresponding Author: Y.-J. Moon a very good correlation for 12 fast halo CMEs. Kim et al.(2008) extended this study by using a much larger database (486 frontside halo CMEs from 1997 to 2003) and more robust statistical tools (contingency table and statistical parameters). They compared the forecast capability of this earthward direction parameter with those of other CME parameters such as location and speed. They found that the direction parameter is superior to other ones and concluded that the CME earthward direction parameter can be an important indicator for forecasting CME geoeffectiveness. On the other hand, setting up CME cone models is a kind of way to identify the geometry and kinematics of a CME. So far there are several different types of cone models (Zhao et al. 2002; Michalek et al. 2003; Xue et al. 2005; Michalek 2006; Zhao 2008).

The present study is one of our series of works supporting the importance of the earthward direction parameter, especially focusing on its geometrical implication and importance. In this study, we present the geometrical meaning of this parameter by deriving the relationship between cone model parameters and the earthward direction parameter. In addition, we demonstrate the consistency between the earthward direction parameters and the cone model parameters for 32 full halo CMEs that Michalek et al.(2003) used. Finally we discuss the advantages of this parameter in terms of space weather forecast. The paper is organized as follows. In section 2, we explain our data and the di- 
rection parameter. We present the results of our study with discussions in section 3 . A brief summary and conclusion are delivered in section 4 .

\section{DATA AND ANALYSIS}

To compare our earthward direction parameter and cone model parameters, we consider front-side full halo CMEs that Michalek et al.(2003) compiled. They developed a cone model to determine the widths, velocities, and source location of these CMEs under an assumption that they have constant velocities, are symmetric, and propagate with constant angular widths. They successfully estimated their cone model parameters (for details see their table 1). They are SOHO/LASCO (Large Angle Spectroscopic COronagraph; Brueckner et al. 1995) CMEs from 1996 to 2000. Their CME properties such as linear plane-of-sky speeds, angular widths, and position angles were collected from the CME online catalogue (http://cdaw.gsfc.nasa.gov/CME_list/index.html; Yashiro et al. 2004). Most of these CMEs are associated with flares and their speeds are much faster than those of normal CMEs (Moon et al. 2002b). Thus they are thought to be very strong potential candidates to produce geomagnetic storms.

If a CME is directly propagating toward the Earth, the shape of the CME front edge should be nearly symmetric. Otherwise, the shape should be quite asymmetric. We thus define the direction parameter, DP, as the ratio of distance between the shorter CME front and the solar center to that of the longer CME front. If a CME has a circular shape, DP would be unity. The earthward direction parameter can be determined directly from a coronagraph observation as follows: (1) an ellipse is plotted to follow the CME front in the SOHO/LASCO running difference image; (2) we then draw a line that passes through the centers of both the Sun and the ellipse formed by the CME front; (3) we measure the ratio of the shorter to longer distance of the CME front from the solar center along this line, and set the ratio as the direction parameter, DP. More detailed descriptions are found in Moon et al.(2005) and Kim et al.(2008). In this way, we could determine the earthward direction parameter values of 32 events and compare them with cone model parameter values. These results are summarized in Table 1.

\section{RESULT AND DISCUSSION}

Figure 1 shows a cone describing the 3 -D shape of a front-side coronal mass ejection. Xie et al.(2004) considered the elliptical projection of the circular crosssection of a CME cone on the plane of sky as shown in Figure 2. According to them, the projection equation of circular cross-section of the cone model on the plane of sky can be described as an ellipse equation:

$$
\left(\frac{x_{c}^{\prime}-h}{a}\right)^{2}+\left(\frac{y_{c}^{\prime}}{b}\right)^{2}=1,
$$

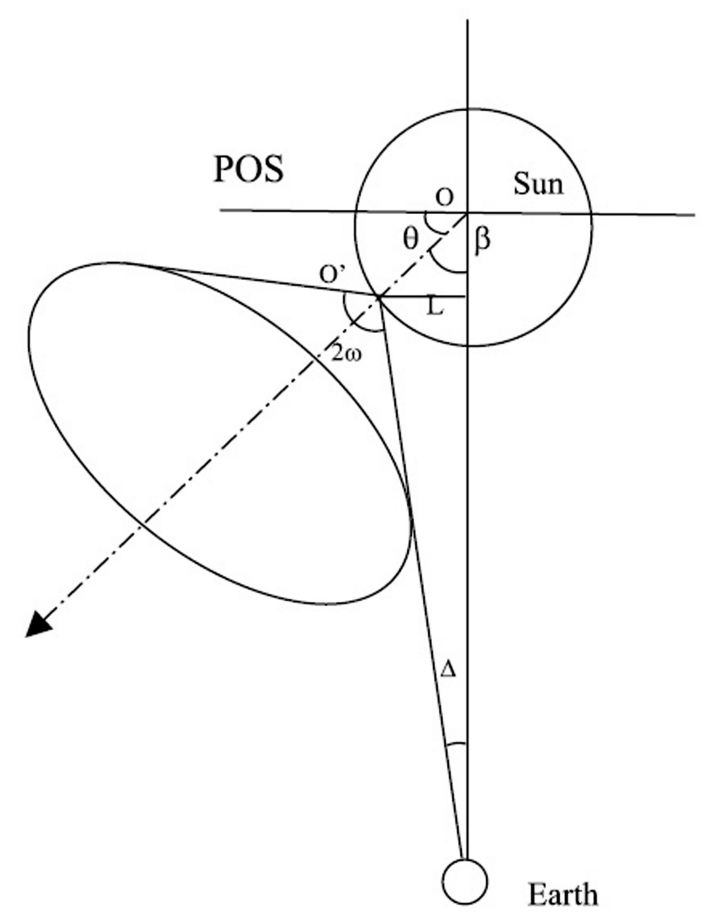

Fig. 1.- Illustration of a front-side CME which ejects from the solar surface to encounter the Earth with $\omega$ the half angular width of a CME cone and $\theta$ the angle between the cone axis and the plane of sky (Xie et al. 2004).

where

$$
h=r \cos \omega \cos \theta, a=r \sin \omega \sin \theta, b=r \sin \omega .
$$

Here the coordinate system $\left(x_{c}^{\prime}, y_{c}^{\prime}, z_{c}^{\prime}\right)$ is for the projected ellipse on the plane of sky, $r$ is the radius from solar center, $\omega$ is the half angular width of the cone, and $\theta$ is the angle between the cone axis and the plane of sky. When $\theta=90^{\circ}$, the CME is a full halo CME which propagates toward the Earth with $\omega$, the half angular width of the cone.

In Figure 2, the projected ellipse corresponds to an ellipse that we considered when estimating the earthward direction parameter. From a direct comparison between the definitions of cone model parameters and the earthward direction parameter DP, we can simply derive the following relationship:

$$
D P=\frac{a-h}{a+h}=\frac{1-\cot \omega \cot \theta}{1+\cot \omega \cot \theta}=-\frac{\cos (\omega+\theta)}{\cos (\omega-\theta)},
$$

where $\cos (\omega-\theta)$ is always positive since $\omega$ and $\theta$ are all between 0 to $90^{\circ}$. If $\theta=0^{\circ}$, then $\mathrm{DP}$ is -1 . If $\theta=90^{\circ}$, then DP is 1 . Here DP is positive when $\omega+\theta>$ $90^{\circ}$ and negative when $\omega+\theta<90^{\circ}$. It is also noted that $\cot \omega \cot \theta$ (i.e., h/a) has a similar concept of the 
TABLE 1.

Summary of SOHO/LASCO Halo CMEs used for COMPARISON.

\begin{tabular}{|c|c|c|c|c|c|}
\hline LASCO Time (UT) & $\theta\left(^{\circ}\right)$ & $2 \omega\left(^{\circ}\right)$ & $\operatorname{Lat}\left({ }^{\circ}\right)$ & $\operatorname{Lon}\left({ }^{\circ}\right)$ & $\mathrm{DP}$ \\
\hline 19970407 14:27:44 & 65 & 139 & -30 & -19 & 0.57 \\
\hline 19971021 18:03:45 & 75 & 103 & 20 & -12 & 0.60 \\
\hline 19971106 12:10:41 & 34 & 153 & -18 & 63 & 0.31 \\
\hline 19980125 15:26:34 & 60 & 114 & 24 & -27 & 0.46 \\
\hline 19980429 16:58:54 & 74 & 111 & -17 & -20 & 0.60 \\
\hline 19980501 23:40:59 & 84 & 40 & -18 & 5 & 0.71 \\
\hline 19980502 05:31:56 & 85 & 39 & -20 & 17 & 0.75 \\
\hline 19980605 12:01:53 & 39 & 159 & -23 & -43 & 0.27 \\
\hline 19981105 02:24:56 & 79 & 88 & 19 & 10 & 0.84 \\
\hline 19981105 20:58:59 & 69 & 75 & 22 & 18 & 0.51 \\
\hline 19981124 02:30:05 & 27 & 153 & -30 & 81 & 0.52 \\
\hline 19981218 18:21:50 & 47 & 120 & 19 & -64 & 0.37 \\
\hline 19990503 06:06:05 & 51 & 110 & 15 & -32 & 0.34 \\
\hline 19980510 05:50:05 & 74 & 76 & 16 & -19 & 0.58 \\
\hline 19990626 07:31:25 & 83 & 67 & 25 & 0 & 0.60 \\
\hline 19990628 12:06:07 & 47 & 143 & -27 & -55 & 0.60 \\
\hline 19990630 11:54:07 & 80 & 92 & -15 & 0 & 0.80 \\
\hline 19990725 13:31:21 & 40 & 127 & 29 & 81 & 0.25 \\
\hline 19991222 02:30:05 & 40 & 162 & 10 & -30 & 0.38 \\
\hline 19991222 19:31:22 & 69 & 141 & 24 & -19 & 0.39 \\
\hline 20000208 09:30:05 & 50 & 162 & 25 & -26 & 0.44 \\
\hline 20000209 19:54:17 & 63 & 128 & -17 & 40 & 0.40 \\
\hline 20000606 15:54:05 & 71 & 152 & 21 & -15 & 0.88 \\
\hline 20000610 17:08:05 & 50 & 138 & 22 & 37 & 0.42 \\
\hline 20000711 13:27:23 & 47 & 162 & 18 & -27 & 0.55 \\
\hline 20000912 11:54:05 & 54 & 159 & -12 & 18 & 0.48 \\
\hline 20000916 05:18:14 & 74 & 126 & 14 & -4 & 0.80 \\
\hline 20001123 06:06:05 & 60 & 150 & -22 & 33 & 0.54 \\
\hline 20001124 05:30:05 & 62 & 147 & 22 & 2 & 0.58 \\
\hline 20001124 15:30:05 & 74 & 96 & 22 & 7 & 0.53 \\
\hline 20001124 22:06:05 & 55 & 130 & 21 & 14 & 0.40 \\
\hline 20001126 17:06:05 & 54 & 144 & 18 & 38 & 0.51 \\
\hline
\end{tabular}

eccentricity of an ellipse. Figure 3 shows the variation of DP as a function of $\omega$ and $\theta$. It is found that DP increases with $\omega$ and $\theta$, with the same dependence on each parameter. As seen in Figure 1, the CME cone has a higher probability to encounter the Earth when either $\theta$ or $\omega$ becomes large. Of course, the larger both values are, the higher the probability is; equivalently, the larger our earthward direction parameter is, the higher the probability is. This fact implies that our earthward direction parameter include both the effects of the cone axis direction $\theta$ and the half angular width $\omega$.

If we consider a self-similar expansion of a CME that is propagating from the Sun to the Earth in Figure 1, the condition for the CME to encounter the Earth is given by

$$
\omega>90^{\circ}-\theta
$$

This relationship is equivalent to $D P>0$ as shown in the above paragraph. This result indicates that if DP of a halo CME is positive, then it has a possibility to encounter the Earth. Of course, the CME encounter the Earth with a higher probability when DP is larger.

Figure 4 shows a comparison between our earthward direction parameter DP and $\theta$, the angle between the cone axis and the plane of sky, for 32 front-side halo CMEs. As seen in the figure, there is a good correlation between two parameters with $\mathrm{r}=0.72$, which is fairly good when considering that our direction parameter depends on both parameters, $\theta$ and $\omega$. If a CME is radially ejected from the solar surface and the CME origin is the same as its associated flare location, then one can derive the angle $\theta$ (flare) from flare location. Figure 5 shows a comparison between $\theta$ (cone) from the cone model fitting and $\theta$ (flare) from the flare location. As seen in the figure, there are noticeable differences 


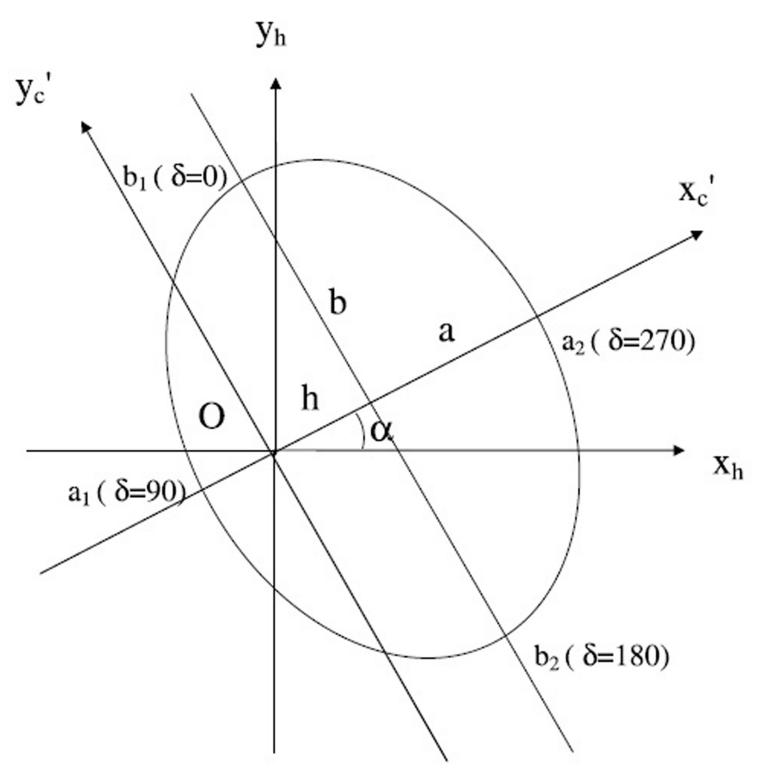

Fig. 2.- Elliptical projection of the circular cross-section of the cone on the plane of sky (Xie et al. 2004). The heliocentric coordinate system $\left(x_{h}, y_{h}, z_{h}\right)$ and the coordinate system for projected ellipse $\left(x_{c}^{\prime}, y_{c}^{\prime}, z_{c}^{\prime}\right)$ are used.

between two parameters, especially for small values of $\theta$. This implies that a CME does not always eject radially from the Sun and $\theta$ (flare) should not be considered as a good direction parameter.

\section{SUMMARY AND CONCLUSION}

In this study, we have presented the geometrical implication of our earthward direction parameter by comparing it with the cone model parameters. Major results from this study can be summarized as follows. First, we have derived an analytic relationship between the cone model parameters (half angular width $\omega$ of the CME cone and the angle $\theta$ between the cone axis and the plane of sky) and the earthward direction parameter DP. This relationship shows that our direction parameter shows the degree of symmetry of the projected ellipse on the plane of sky and it is closely related to the eccentricity of the ellipse. Second, the plot of DP as a function of $\omega$ and $\theta$ shows that DP increases with $\omega$ or $\theta$ and has the same dependence on each parameter. This implies that our earthward direction parameter include both the effects of the cone axis direction and the cone angular width. This fact supports that the earthward direction parameter should be superior to other individual parameters, which were shown in Kim et al.(2008). Third, we have demonstrated the close correlation between the earthward direction parameter and the cone axis direction $\theta$ using 32 front-side full halo CMEs. Its correlation value is 0.72 , which is relatively good when

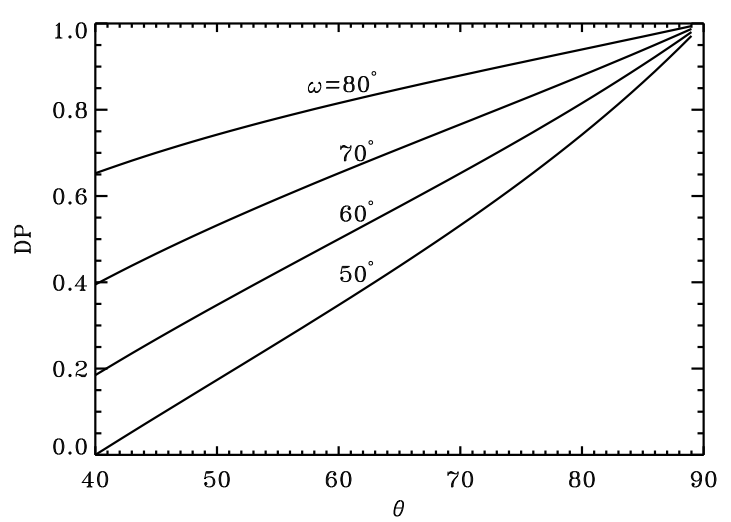

Fig. 3.- Relationship between the earthward direction parameter DP and the cone model parameters, $\omega$ and $\theta$.

considering that our direction parameter depends on $\theta$ as well as $\omega$. Fourth, we have also found that there are noticeable differences between two values of $\theta$ from the cone model fitting and from the solar flare position, implying that flare position should not be used as a good direction parameter.

Our earthward direction parameter has several strong advantages in terms of the forecast of a geomagnetic storm based on CME parameters. First, it is directly observable from coronagraph observations by a simple ellipse fitting to the CME front and additional distance measurements. Second, it is applicable to most of the halo CMEs, even for the event whose flare location is not known. This characteristic was demonstrated by Kim et al.(2008) who estimated the earthward direction parameters of most of the front-side halo CMEs from 1997 to 2003. It is also noted that the cone model of Michalek et al.(2003) could be applied to about one third of the halo CMEs. Third, we have no special assumption of the CME shape such as a specific cone shape. Fourth, this parameter includes both the effects, cone axis direction $(\theta)$ and angular width $(\omega)$, that are two key parameters to control the encounter of a CME to the Earth. Moreover, our earthward direction parameter is superior to other parameters such as CME speed, CME position, magnetic field orientation in a CME source region, as demonstrated in Kim et al.(2008).

\section{ACKNOWLEDGEMENTS}

We appreciate the referee's careful reading and suggestions. This study was motivated by the anonymous referee of Moon et al.(2005). This work has been supported by the WCU grant (No. R31-10016) funded by the Korean Ministry of Education, Science and Technology and by the Korea Research Foundation Grant funded by the Korean Government (MOEHRD, Basic 


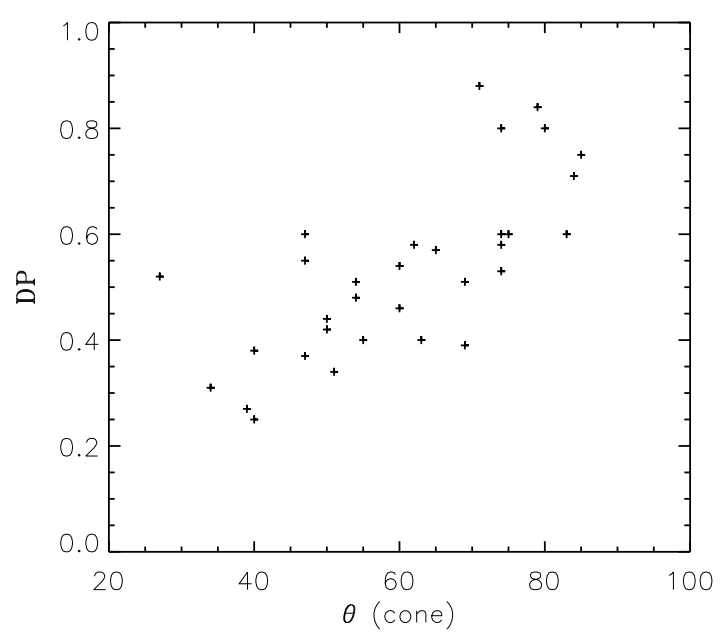

Fig. 4.- Relationship between the earthward direction parameter DP from this study and $\theta$ the angle between the cone axis and the plane of sky for 32 front-side halo CMEs.

Research Promotion Fund) (KRF-2008-314-C00158). CKS has been supported by the Ministry of Science and Technology (MOST) grant (M1-0407-00-0001) of the Korean government, the "Development of Korean Space Weather Center" project of KASI, and the KASI basic research fund.

The CME catalogue we have used is generated and maintained by the Center for Solar Physics and Space Weather, The Catholic University of America in cooperation with the Naval Research Laboratory and NASA. SOHO is a project of international cooperation between ESA and NASA.

\section{REFERENCES}

Brueckner, G. E., et al., 1995, The Large Angle Spectroscopic Coronagraph (LASCO), Solar Phys., 62, 357

Cho, K.-S., Moon, Y.-J., Dryer, M., Fryer, C. D., Park, Y.-D., \& Kim, K.-S., 2003, A Statistical Comparison of Interplanetary Shock and CME Propagation Models, J. Geophys. Res., 108, 1445

Gopalswamy, N., Yashiro, S., \& Akiyama, S. 2007, Geoeffectiveness of Halo Coronal Mass Ejections, J. Geophys. Res., 112, A06112, doi:10.1029/2006JA012149

Kang, Seung-Mi, Moon, Y.-J., Cho, K.-S., Kim, YeonHan, Park, Y.-D., Baek, Ji-Hye, \& Chang, HeonYoung, 2006, Coronal Mass Ejection Geoeffectiveness depending on Field Orientation and Interplanetary Coronal Mass Ejection Classification, J. Geophys. Res., 111, doi:10.1029/2005JA011445

Kim, K.-H., Moon, Y.-J., \& Cho, K.-S., 2007, Prediction of the 1-AU Arrival Times of CME-associated

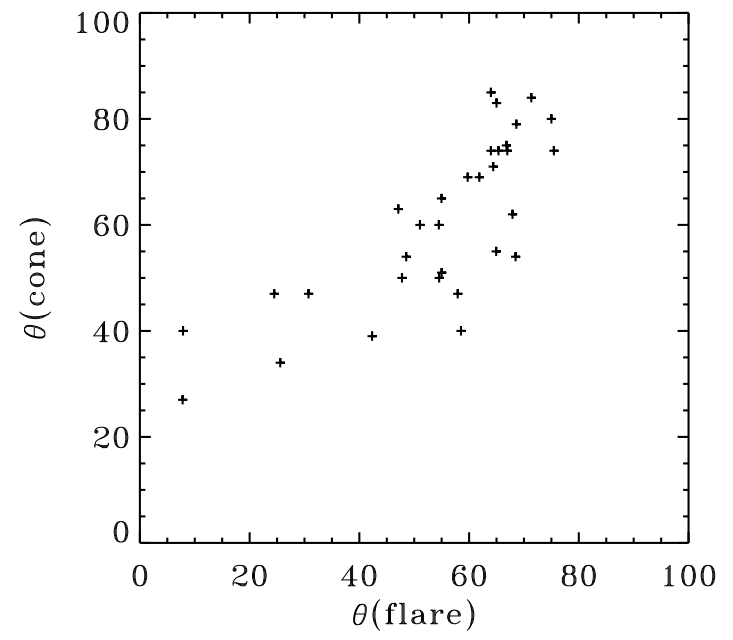

Fig. 5.- Relationship between the angle $\theta$ (cone) from the cone model fittings and the angle $\theta$ (flare), which is estimated under the assumption that a CME was ejected radially from its associated flare location.

Interplanetary Shocks: Evaluation of an Empirical Interplanetary Shock Propagation Model, J. Geophys. Res., 112, A05104, doi:10.1029/2006JA011904

Kim, R.-S., Cho, K.-S., Moon, Y.-J., Kim, Y.-H., Yi, Y., Dryer, M., Bong, Su-Chan, \& Park, Y.D., 2005, Forecast Evaluation of the Coronal Mass Ejection (CME) Geoeffectiveness using Halo CMEs from 1997 to 2003, J. Geophys. Res., 110, A11104, doi:10.1029/2005JA011218

Kim, R.-S., Cho, K.-S., Kim, K.-H., Park, Y. D., Moon, Y.-J., Yi, Y., Lee, J., Wang, H., \& Song, H., 2008, CME Earthward Direction as an Important Geoeffectiveness Indicator, ApJ, 677, 1378

Michalek, G., Gopalswamy, N., \& Yashiro, S., 2003, A New Method for Estimating Widths, Velocities, and Source Location of Halo Coronal Mass Ejections, ApJ, 584, 472

Michalek, G., 2006, An Asymmetric Cone Model for Halo Coronal Mass Ejections, Sol. Phys., 237, 101

Moon, Y.-J., Dryer, M., Smith, Z., Park, Y.-D., \& Cho, K. S., 2002a, A Revised Shock Time of Arrival(STOA) Model for Interplanetary Shock Propagation: STOA-2, Geophys. Res. Lett., 29,

Moon, Y.-J., Choe, G. S., Wang, H., Park, Y. D., Gopalswamy, N., Yang, G., \& Yashiro, S., 2002b, A Statistical Study of Two Classes of Coronal Mass Ejections, ApJ, 581, 694

Moon, Y.-J., Cho, K.-S., Dryer, M., Kim, Y.-H., Bong, Su-Chan, Chae, Jongchul, \& Park, Y. D., 2005, New Geoeffective Parameters of Very Fast Halo Coronal Mass Ejections, ApJ, 624, 414 
Xie, H., Ofman, L., \& Lawrence, G., 2004, Cone Model for Halo CMEs : Application to Space Weather Forecasting, J. Geophys. Res., 109, A03109, doi:10.1029/2003JA010226

Xue, X. H., Wang, C. B., \& Dou, X. K., 2005, An icecream cone model for coronal mass ejections, J. Geophys. Res., 110, A8, CiteID A08103

Yashiro, S., Gopalswamy, N., Michalek, G., Rich, N., St.Cyr, C. O., Plunkett, S. P., \& Howard, R. A., 2004, A Catalog of White Light Coronal Mass Ejections Observed by the SOHO Spacecraft, J. Geophy. Res. 109, A7, A07105

Zhao, X. P., Plunkett, S. P., \& Liu, W., 2002, Determination of geometrical and kinematical properties of halo coronal mass ejections using the cone model, J. Geophys. Res., 107, A8, pp. SSH 13-1, CiteID 1223, doi:10.1029/2001JA009143

Zhao, X. P., 2008, Inversion solutions of the elliptic cone model for disk frontside full halo coronal mass ejections, J. Geophys. Res., 113, A2, CiteID A02101 